\section{Cahiers de Narratologie}

Analyse et théorie narratives

$28 \mid 2015$

Le récit comme acte cognitif

\title{
Les comédiens de stand-up et la preuve par le rire : le récit comme acte cognitif dans Star Wars Canteen $1 \& 2$ d'Eddie Izzard*
}

\section{Guillemette Bolens}

\section{(2) OpenEdition}

Journals

Édition électronique

URL : https://journals.openedition.org/narratologie/7187

DOI : 10.4000/narratologie. 7187

ISSN : 1765-307X

Éditeur

LIRCES

Référence électronique

Guillemette Bolens, "Les comédiens de stand-up et la preuve par le rire : le récit comme acte cognitif dans Star Wars Canteen 1 \& $2 d^{\prime}$ Eddie Izzard* », Cahiers de Narratologie [En ligne], 28 | 2015, mis en ligne le 09 juillet 2015, consulté le 11 juin 2021. URL : http://journals.openedition.org/narratologie/7187 ; DOI : https://doi.org/10.4000/narratologie.7187

Ce document a été généré automatiquement le 11 juin 2021.

Article L.111-1 du Code de la propriété intellectuelle. 


\title{
Les comédiens de stand-up et la preuve par le rire : le récit comme acte cognitif dans Star Wars Canteen 1 \& 2 d'Eddie Izzard*
}

\author{
Guillemette Bolens
}

\section{NOTE DE L'AUTEUR}

*L'écriture de cet article s'inscrit dans le cadre d'un projet de recherche financé par le Fonds National Suisse de la Recherche Scientifique intitulé « Kinesic Knowledge in Anthropology and Literature » (Division I, n¹00016_150264), mené sous la direction de l'auteur.

1 Le spectacle de 2013 de l'humoriste britannique Eddie Izzard porte le titre de Force Majeure et contient une continuation de l'un de ses sketchs les plus connus, Star Wars Canteen (ou Death Star Canteen ${ }^{1}$ ). Dans la version filmée qui se trouve sur YouTube, la caméra se tourne régulièrement vers les spectateurs, permettant d'observer la relation étroite qui s'établit entre l'artiste et son audience à travers un récit performé, une coprésence inter-réactive, et la connaissance d'un répertoire culturel partagé. En effet, la compréhension des sketchs Star Wars Canteen $1 \& 2$ se fonde, dans les deux cas, sur la connaissance du monde fictionnel (storyworld) des films de George Lucas, Star Wars, fleuron d'une culture populaire massivement amplifiée à travers différents média. Le récit performé dans Force Majeure donne l'occasion d'observer, en outre, une relation cognitive en acte entre le raconteur (storyteller) et son audience. Car certains éclats de rire impliquent une saisie cognitive remarquablement rapide du lien qu'établissent les énonciations d'Eddie Izzard avec son premier sketch, Star Wars Canteen 1, créé en 2000 dans son spectacle Circle. Cet acte cognitif du spectateur en réponse à l'acte cognitif 
puis énonciatif du raconteur donne lieu à une manifestation de nature kinésique, l'éclat de rire'2.

Des humains écoutent un autre humain avec une attention soutenue et deviennent soudain le lieu d'un mouvement complexe et saccadé de tout le torse, accompagné parfois par une projection rapide de la tête vers l'arrière, la bouche s'ouvrant davantage tandis que les yeux se ferment brusquement. La raison de cet événement kinésique, que nous connaissons tous d'expérience, est ce qui s'appelle l'éclat de rire. Or, il a pour condition sine qua non un événement cognitif. Il n'y a aucun contact tactile entre l'humoriste et les spectateurs (il ne les chatouille pas) et ses mouvements corporels ne sont pas comiques en eux-mêmes. L'éclat de rire n'aurait pas lieu sans la parole. Un comédien ou une comédienne de stand-up est une personne qui, comme son nom l'indique, se tient debout devant des spectateurs et parle - pour les faire rire : c'est ce qui le distingue d'un politicien ou d'un professeur (distinction qui peut éventuellement se limiter à l'intention de l'orateur). Le résultat cognitif généré par le sketch initial d'Izzard concerne une situation que le spectateur ou la spectatrice n'avait a priori jamais envisagée jusqu'au moment où Izzard l'énonce, à savoir celle du personnage de Darth Vader commandant des pâtes à la cafétéria de la station spatiale appelée Étoile de la mort, The Death Star. Le sketch permet d'observer un avant et un après cognitif de cette situation inconcevable dans le monde fictionnel des films originaux de Lucas.

3 Le genre du stand-up se construit sur le rapport que l'humoriste établit avec son public. Il ou elle réagit aux réactions des spectateurs après les avoir provoquées. Dans sa définition du stand-up, Chris Ritchie écrit qu'il s'agit généralement d'une performance en solo avec microphone, dont le succès ne se mesure pas au nombre de personnes dans la salle mais à la qualité de la relation que l'artiste parvient à établir avec les personnes présentes (Ritchie 2012: 12). Typiquement, Eddie Izzard renforce cette relation et la rend manifeste au moment où il fait un commentaire sur les rires provoqués par son dernier gag, avant de se lancer dans le sketch de Darth Vader à la cantine. La notion d'acte dans l'idée d'acte cognitif se situe doublement chez le comédien, qui imagine un récit, puis agit par lui sur la cognition de ses spectateurs. Mais il se situe également chez le spectateur lui-même, qui s'engage dans cette interaction dès lors qu'il écoute activement l'humoriste et prête attention à son jeu narratif. Il s'agit donc d'une activité réciproque, laquelle semble suffisamment gratifiante pour que l'un en fasse son métier et l'autre paie pour en bénéficier.

Or donc Darth Vader va à la cantine et commande des penne all'arrabbiata. Car, nous dit Izzard, entre deux batailles, il fallait bien qu'il mange. L'employé qui fait le service demande "Will you need a tray?». Et il s'entend répondre d'une voix gutturale et menaçante, « Do you know who I am ? ». Cet échange fonctionne aujourd'hui comme une référence humoristique immédiatement identifiée par les nombreux fans d'Izzard et fait partie du répertoire culturel des adeptes de stand-up. Tout le sketch se construit sur le hiatus qui sépare le registre prosaïque et pragmatique d'un service en cantine (il vous faut un plateau car les assiettes sont chaudes) et les prétentions suprémacistes et mégalomaniaques du seigneur de la Death Star, lequel clame bientôt :

-I don't need a tray. I can kill you with a single thought.

-You will still need a tray.

-No, I will not need a tray. I do not need a tray to kill you. I can kill you without a

tray with the power of the Force, which is strong within me. Even though I could 
kill you with a tray, if I so wished, for I would hack at your neck with the thin bit until the blood flowed upon the canteen.

- No, the food is hot. You need a tray to put the food on.

-Oh I see, the food is hot. I'm sorry. I did not realize.

5 Ce n'est que le début du sketch, et il y a déjà beaucoup à en dire. Je choisis de me concentrer sur deux aspects: le récit transmédial (transmedial storytelling) et la dimension kinésique de la narration en conversation (narrative in conversation).

\section{Le récit transmédial dans le stand-up posté sur YouTube}

Dans Storyworlds across Media, Marie-Laure Ryan souligne qu'un même monde fictionnel (storyworld) peut se déployer dans de nombreux récits différents (Ryan 2014 : 41). C'est le cas dans les cultures orales, où les poètes répètent et amplifient un fond narratif connu de leur audience. C'est également vrai du phénomène actuel appelé media convergence ou transmedial storytelling (Ryan $2014: 41$ ). Ce phénomène peut se réaliser par de multiples auteurs, canoniques ou apocryphes, qui développent le monde fictionnel d'un film ou d'un roman populaire comme The Lord of the Rings, Harry Potter ou Star Wars (Ryan 2014 : 41). Les deux sketchs d'Izzard peuvent être considérés comme des cas de transmédialisation, puisque le monde fictionnel de Star Wars est développé par un artiste apocryphe, l'humoriste Eddie Izzard, passant du film de cinéma grand public à la scène de spectacle en direct, dont les séquences sont ensuite postées sur YouTube et visionnées au moyen d'Internet sur un ordinateur ${ }^{3}$.

7 Ryan explique que «les éléments variés d'un système transmédial peuvent soit déployer un monde fictionnel à travers des procédés qui respectent le contenu précédent, soit créer des mondes fictionnels distincts du point de vue logique tout en étant liés par l'imaginaire, grâce à des modifications et des transpositions qui altèrent le contenu existant » (Ryan 2014 : 42, TdA). En ce qui concerne Star Wars, les six films de George Lucas représentent le même monde, alors que les divers jeux informatiques, mondes en ligne, romans et textes inspirés par les films altèrent ce monde fictionnel de multiples façons (Ryan 2014 : 42). Les sketchs d'Izzard altèrent eux aussi le storyworld de Star Wars. Darth Vader n'est plus seulement un méchant très dangereux qui fait du bruit en respirant, c'est un personnage à qui il arrive d'avoir faim et qui va à la cantine manger des pâtes à la sauce pimentée.

8 Les altérations effectuées par Izzard sont cohérentes vis-à-vis de ce que Chris Ritchie appelle le microworld du comédien de stand-up. Chaque artiste de stand-up crée à travers ses sketchs une certaine vision du monde, son microworld. L'image de ce microworld se construit graduellement, sketch après sketch, chaque blague augmentant notre compréhension de la manière de voir de l'humoriste et surtout de sa façon de ressentir les choses (Ritchie 2012 : 38). Dans le microworld propre au comédien Izzard, même le très méchant qui n'enlève jamais son masque doit tôt ou tard avoir envie de faire une pause et de manger un petit quelque chose. Certes, il choisira alors une sauce piquante dont le nom évoque la colère, mais enfin c'est bien qu'il aura faim de temps en temps. L'humour d'Izzard souvent démystifie les gesticulations de toute-puissance par une intervention prosaïque du quotidien.

9 Pour Ryan, l'un des objectifs d'une narratologie attentive au médium (media-conscious narratology) est de décrire les relations qui existent entre les mondes des systèmes 
transmédiaux, et de fournir des critères permettant de distinguer entre les représentations d'un même monde fictionnel et les cas où l'amplification projette un monde lié mais distinct du monde fictionnel initial (Ryan 2014: 42). Dans les pratiques de transmedia storytelling, Jason Mittell distingue celles qui relèvent de l'établissement de faits complémentaires, correspondant à la question What Is ?, de celles qui élaborent des variantes hypothétiques, correspondant à la question What If ? (Mittell $2014: 273$ ). Le nouveau film de Star Wars, annoncé récemment et en cours de tournage en 2015, relèvera clairement du What Is. Par contre, Death Star Canteen d'Izzard répond à la question What If? En même temps, le récit de la cafétéria commence par une déclaration de fait : "The one thing about the Death Star is that there was no food, no one had food, no food at all ». Pour amplifier cette réponse au What Is? (-Did they have any food? No!), Izzard performe un échange fictionnel entre collègues de travail, où l'un d'eux, s'apprêtant à passer à l'épicerie du coin, demande à Darth Vader ce qu'il veut, un sandwich jambon ou poulet? Mais c'est pour aussitôt nier cette éventualité puisqu'il n'y a justement pas de nourriture sur la Death Star. Et c'est alors seulement que l'humoriste lance la scène de la cantine, en réponse à la question implicite de type What If : et s'ils avaient faim? «But there must have been a Death Star canteen, yeah ?, there must have been a cafeteria downstairs, in between battles, where Darth Vader could just chill and go down. "-I will have the penne all'arrabbiata!" ». Le What If domine alors, mais après s'être embranché sur un établissement des faits de type What Is (no food at all).

Selon Mittell, les transmedia qui relèvent du What Is cherchent à développer la fiction de manière cohérente selon un canon, en expliquant l'univers narré aussi précisément que possible, dans l'objectif d'augmenter la compréhension et l'appréciation du monde fictionnel en question (Mittell 2014 : 273). En revanche, le mode transmédial du What If vise d'autres buts narratifs et signale sa réussite différemment. Il s'agit d'envisager " des possibilités hypothétiques plutôt que des certitudes canoniques, en invitant les spectateurs ou destinataires (viewers) à imaginer des histoires alternatives et des approches de l'acte de raconter (storytelling) qui soient clairement à traiter autrement que comme la source d'un canon potentiel ». Le but des transmédias du What If est « de lancer le récit source dans des dimensions parallèles, avec des connections qui mettent en avant des questions de ton, de modalité, de personnage, ou de style » (Mittell 2014 : $273, \mathrm{TdA})$.

11 Le ton, la modalité et le style des sketchs d'Izzard appartiennent de toute évidence à une dimension parallèle, non pas seulement en raison du remarquable style vestimentaire de l'humoriste - et quand bien même Darth Vader en pantalons de cuir moulant, en soi, mérite le détour ${ }^{4}$. Plutôt, l'une des conséquences notables de l'approche What If de ces sketchs est de modifier radicalement le ton et la modalité de ce monde fictionnel transmédialisé et d'y introduire de nouveaux personnages, tel l'employé de cantine, mais aussi Mister Stephens. Dans le numéro original, la seule évocation de Mister Stephens suffit à déclencher le rire, pour des raisons que nous allons voir. Puis, dans la suite du sketch, en 2013, Mister Stephens joue un rôle clé dans l'action elle-même et dans les dialogues. Afin de pouvoir réfléchir à cette conséquence du transmédia de type What If dans le récit performé de stand-up, nous allons considérer de plus près la notion de narration en conversation. 


\section{Le joke et la narration en conversation}

12 Le genre du stand-up peut être défini comme une conversation performée entre le comédien et les spectateurs - avec des nuances, selon les artistes ${ }^{5}$. Certains posent des questions aux membres du public (par exemple Russell Peters), d'autres improvisent sur leurs réactions. Un humoriste comme Ed Aczel fait délibérément échouer sa relation avec les spectateurs en posant à ceux-ci des questions de façon mécanique à partir de notes qu'il lit sous leurs yeux. Dans tous les cas, le quatrième mur est absent, contrairement à la scène théâtrale, où la performance se déroule a priori sans l'intrusion de la salle dans l'intrigue, et réciproquement. Selon Ritchie, « La comédie en direct est le seul mode de performance dans lequel l'audience peut intervenir et affecter le spectacle directement" (Ritchie 2012: 16, TdA). C'est là en soi un défi important pour les humoristes, qui doivent montrer une capacité virtuose à exploiter l'imprévu, en l'intégrant de façon naturelle dans leur performance. C'est aussi un cas de figure de premier intérêt pour la relation entre énonciation linguistique et acte cognitif entre interlocuteurs.

13 Le comédien de stand-up s'adresse aux personnes présentes (parfois en les tutoyant), parle à la première personne du singulier et fait référence à sa propre vie. Il ou elle raconte ce qui lui est arrivé. Cette approche autobiographique a été initiée aux EtatsUnis par Mort Sahl et Lenny Bruce dans ce qui était considéré comme de la comédie alternative dans les années 60 et qui maintenant domine le champ du spectacle humoristique (Ritchie 2012 : 1, 4). Même lorsque le spectacle ne s'inscrit pas stricto sensu dans un récit de vie (véridique ou fictionnel), les références personnelles ou pseudopersonnelles structurent le spectacle. Par elles, le comédien de stand-up construit ce que Ritchie appelle son persona, à savoir l'identité qu'il présente sur scène. Il ne s'agit pas d'un personnage mais du «vrai soi » de l'artiste, augmenté ou exagéré («the performer's "real self" heightened or exaggerated ») (Ritchie $2012: 11, \mathrm{TdA}$ ).

14 Florence Foresti a libéré par son seul spectacle Mother Fucker (2009-2010) la façon de parler de la grossesse, de l'accouchement et de la maternité. Tout prête à penser - et elle le déclare elle-même - qu'elle a pu le faire grâce à son expérience personnelle. Mais les détails d'authenticité autobiographique servent principalement à ancrer les extrapolations humoristiques de l'artiste dans un vécu concret qui augmente l'efficacité de ses sketchs. Elle aussi s'adresse systématiquement aux spectateurs, par exemple quand elle lance le sketch de la poussette avec la question, «Qu'est-ce qu'on fait, on en parle de la poussette ou pas? Non ça va? Ça dérange personne, tout va bien? J'veux dire, on est en 2010, on fait du feu avec un iPhone, mais on peut toujours pas plier cette merde! ». La complicité et la connaissance partagée établissent une intersubjectivité, qui, selon les termes de Keith Murphy, crée "une union phatique entre interlocuteurs " (Murphy 2011: 245, TdA). Le rire du public est crucial à la poursuite de cette sorte de conversation et prouve de façon sonore et constante qu'une telle union phatique est en cours. Bill Burr, à travers un microworld radicalement différent, vise le même effet quand il demande aux spectateurs s'ils veulent qu'il leur raconte une dispute qu'il a eue avec son amie : "You guys want to hear that story? You want to hear that one? This is what happened ${ }^{6} »$. Compte avant tout le sentiment de sincérité partagée -sentiment qui conditionne la réception du récit dans le cadre de cette conversation performée entre artiste et public. 

façon autobiographique à son propre père, soit " a punch of the bracket ». " A punch of the bracket » est un coup de poing en pleine face. Izzard emploie cette expression après avoir imité un personnage ridicule de Star Wars " who needs a punch of the bracket ». Il explique le sens de cette expression par des gestes et ajoute, «I don't know, my dad used to say it to me, "-You need a good punch of the bracket" ». Puis il rectifie : " No, he didn't say that. He said "-I'll smash you to that wall" ». Et l'audience éclate de rire. Ce moment du spectacle est construit sur la structure de base d'une blague (joke), d'après Greg Dean? Une blague se construit sur une première ligne narrative (1st story) qui crée une anticipation chez le spectateur, puis sur une deuxième ligne narrative $\left(2^{\text {nd }}\right.$ story) qui vient contredire cette assomption et produire un effet de surprise (Dean $2000: 3$ ). La rectification (non, mon père ne me disait pas qu'il allait me frapper en pleine face) fait anticiper un père moins pathologique. Le rire est déclenché par la surprise d'une rectification qui en fait augmente massivement la violence impliquée. Morreall parle de «cognitive shift » pour faire référence à l'effet cognitif du passage de l'anticipation préparée à sa rectification surprenante (Morreall 2009 : 50-52). suffire de deux phrases. Par ailleurs, on voit que le genre du stand-up emploie le récit (pseudo)-autobiographique pour faire rire sur des sujets parfois révoltants, en actionnant le mécanisme cognitif de la surprise - ce qui soulève le problème de la responsabilité de l'artiste ${ }^{8}$. Chez Izzard, la violence du propos est canalisée par le fait que son persona parle de lui-même et que son élocution suggère que l'exagération surprenante est un acte fictionnel de sa part. L'information personnelle est déviée vers un jeu linguistique, où le rapport entre l'action d'un père pathologique menaçant son enfant, devient le lieu d'un souci de précision référentielle : que signifie l'expression punch of the bracket et quelle était exactement l'expression utilisée. En outre, la blague montre un père pouvant adopter des postures verbales de toute-puissance montrées comme absurdes. La violence apparait comme ridicule, risible, et sa nocivité est dégonflée. À cela s'ajoute enfin la dégaine de l'artiste, à la fois énergique et mesuré, le sourire en coin. Le rire du public n'implique pas une perversité sadique de la part des spectateurs, mais une efficacité humoristique de la part de l'artiste, qui sait manier la surprise narrative9.

17 Après la digression du punch of the bracket, Izzard revient à Star Wars et explicite cette redirection de la conversation: «Umm, where was I going?, yes, yes, yes, so eeer Stars Wars, I mean the Death Star, the Death Star is almost like a New York name ». Pour expliquer ce qu'il entend par là, Izzard insère, dans sa conversation avec le public, une micro narration qui a elle-même la forme d'une conversation. Celle-ci a lieu entre des personnages anonymes.

The Death Star is almost like a New York name. The Death Star! Get to the point!

-What's that star?

-The Death Star.

-What does it do?

-It does death! [Intensification du ton] IT DOES DEATH, BUDDY! Get out of my way!

Izzard incarne le premier et le second personnages en se tournant d'un côté puis de l'autre, et en modifiant le ton de sa voix. Le contenu du récit enchâssé de cette interaction minimale est pertinent de façon très relative au niveau linguistique et propositionnel (ce qui fait partie de la blague: l'explication n'explique rien). Cependant, son efficacité humoristique performée est considérable, principalement en 
raison du changement de ton et de kinésie dans le passage d'une phrase à l'autre, évoluant en une fraction de seconde d'une question naïve et neutre à une réponse brusquement agressive. Tout comme la digression du punch of the bracket, cette dynamique narrative, basée sur un effet de surprise cette fois kinésique, crée une atmosphère qui sert de prologue à la partie centrale du sketch. Car ces trois moments (l'expression employée par le père ; le dialogue anonyme ; Darth Vader à la cantine) ont pour dénominateur commun le récit performé d'une attitude dont l'agressivité fait rire tant elle paraît décalée et absurde.

Lorsque Darth Vader comprend pourquoi l'employé de la cantine lui demande s'il a besoin d'un plateau (the food is hot), il explique :

-I thought you were challenging me to the fight to the death.

-Fight to the death? This is canteen. I work here.

-Yes but I am Vader. Lord Vader. Anyone challenges me to a fight to the death [...]

The Death Star. I run the Death Star.

-What's the Death Star?

-This is the Death Star. You're in the Death Star. I run this star.

-This is a star?

-This is a fucking star! I run it. I'm your boss.

-You're Mister Stephens?

-No I'm... Who's Mister Stephens?

- He's head of catering ${ }^{10}$.

-I'm not head of catering! I'm Vader! I can kill catering with a thought!

-What?

-I can kill you all, I can kill me with a thought. Just... Fuck, I'll get a tray, fuck it.

Le décalage entre l'agressivité de Darth Vader et le prosaïsme de l'employé est tel que la violence du premier finit par céder, et il va chercher son plateau : l'épreuve du réel dans toute sa splendeur (the food is hot, get a tray!) au sein du récit d'une conversation enchâssée dans une performance de stand-up au sujet d'une fiction transmédiale (Star Wars).

21 L'humour du sketch au niveau linguistique est fort, il se base sur un hiatus entre registres de pertinence (Sperber \& Wilson 1995). Mais ce niveau n'est qu'une partie d'un ensemble plus complexe, où Izzard montre le hiatus cognitif qui sépare le monde mental inféré de Darth Vader de celui de l'employé de cantine, grâce à la précision et au naturel remarquables de ses intonations vocales, de ses expressions kinésiques et de son timing discursif. La narration de cette interaction entre Darth Vader et l'employé de la cantine est construite sur un humour incarné.

Une manière possible d'en rendre compte est de réfléchir en termes d'expérience narrative, suivant les propositions de Keith Murphy: «En orientant nos perspectives vers ce qui dans une narration crée de l'expérience (the experience creating aspect of narrative), par contraste avec ce qui rend compte de l'expérience (the experience recounting aspect), aspect qui a dominé jusqu'à présent les études narratives, nous serons mieux équipés pour raffiner nos manières d'examiner le rôle que jouent les narrations dans la construction, le maintien et l'interprétation de la réalité sociale " (Murphy 2011: 251, TdA). Pour Murphy, les « analyses structurales d'un récit sont utiles pour mettre en évidence les paramètres formels à travers lesquels les narrations passent dans un univers langagier, mais elles tendent à privilégier le récit lui-même au détriment des personnes qui sont responsables de son apparition dans le monde, d'une part, et des effets particuliers qu'une narration peut avoir sur le monde, d'autre part » (Murphy ${ }^{11}$ 2011: 244). 

" incorporent les récits dans un large éventail de pratiques, employant la narration comme une stratégie de résolution de problème (problem-solving strategy) dans de nombreux contextes " (Herman 2003 : $163 \mathrm{TdA}$ ). De façon semblable, Murphy propose que la narration soit décrite comme une activité, ayant des effets fonctionnels particuliers dans la vie sociale. En effet, l'acte narratif façonne les événements d'une manière qui permet d'organiser leur pertinence, et ce non seulement pour celui qui parle mais aussi pour ses interlocuteurs. L'acte narratif offre l'occasion d'une expérience partagée par le fait même de la narration (Murphy 2011 : 244). Ceci est vrai dans une multitude de contextes, allant du récit d'expériences traumatiques en situation thérapeutique jusqu'à la narration de sketchs dans une comédie de stand-up, en passant par la discussion entre proches et les multiples situations interactionnelles étudiées en sociolinguistique multimodale ${ }^{12}$.

Pour développer des moyens d'analyse de la narration comme activité, il est important d'ouvrir le champ d'observation. Les analyses strictement linguistiques ne peuvent pas suffire qui laisseraient dans l'ombre les qualités incarnées de la narration en conversation (" the embodied qualities of conversational narrative »). Il est nécessaire de porter notre attention sur l'échange parlé, comme les histoires racontées pendant une conversation ne sont pas simplement dites verbalement lors de l'interaction (« not merely told in interaction »), elles sont également démontrées de manière plutôt complexe à travers de multiples canaux communicationnels (« they are also demonstrated in rather complex ways through multiple communicative channels ») (Murphy 2011:245, TdA). Ces démonstrations en narration sont une réalité omniprésente des numéros de stand-up. habituellement le seul objet disponible sur scène dans les spectacles de stand-up. Izzard exploite les possibilités de cet artefact dans sa continuation de la Death Star Canteen en 2013, quand il commence par incarner Dieu en plaçant le micro très près de sa bouche et en l'entourant de ses deux mains. Il affirme que nous souhaitons que Dieu ait une certaine sorte d'élocution. Izzard nous démontre alors ce qu'il entend par là en produisant grâce au micro des sons d'inspire et d'expire excessivement audibles, accompagnés d'une voix caverneuse. Or, ces sons le poussent du côté de la plongée sous-marine. Dieu annonce alors qu'il a commencé à prendre des leçons de plongée (scuba diving) parce qu'il aime regarder passer les petits poissons jaunes - information qu'il démontre illico avec une main opérant doublement et simultanément comme déictique pour désigner un poisson qui passe et comme poisson nageant rapidement à proximité.

En conversation, une démonstration par imitation et/ou utilisation d'artefact (l'inspireexpire au micro) ou une démonstration gestuelle, dans le cas du poisson qui à la fois passe et se trouve désigné par un unique mouvement de la main, peut souvent avoir des implications qui lui sont propres et orienter ainsi le développement de l'échange. Évidemment, le passage de l'élocution divine à la plongée sous-marine en raison des sons produits par l'artefact du microphone est ici planifié par Izzard, mais il rejoue néanmoins le genre d'associations d'idées qui émergent en situation réelle à partir d'une explicitation kinésique d'idées ou d'états mentaux. Les spectacles de stand-up passent souvent d'un sujet à l'autre en exploitant ce genre de connections parfois ténues mais suffisantes. Les gestes donnent des idées. 

du récit comme acte cognitif. En effet, Dieu dit, « Anyway, now I am hungry, I must go to the canteen to get some food ». Après quelques secondes, les spectateurs réagissent avec enthousiasme. Ce laps de temps correspond au processus cognitif par lequel ils font le lien entre l'information «I must go to the canteen » et le sketch original de la Death Star Canteen de 2000. Ce laps de temps est une actualisation perceptible de la durée de l'acte cognitif par lequel l'audience comprend le lien suggéré par l'humoriste. Izzard vient de mettre en place le monde fictionnel (storyworld) de son précédent sketch. Son action discursive a été suivie par son auditoire, dont l'acte cognitif a eu lieu en temps réel.

L'employée de la cantine est cette fois une femme, qui énumère les plats proposés. La dernière assiette désignée comporte des spaghettis alla carbonara. Une seconde de retard sépare "spaghettis alla » de "carbonara». C'est une infime nuance de timing discursif, qui pourtant provoque le rire. Izzard joue avec l'anticipation du public (les pâtes originales étaient des penne all'arrabbiata). Par ce bref retard, il met en évidence l'anticipation cognitive des spectateurs, soulignant par la même occasion la relation qu'il établit en continu avec eux et la connaissance qu'ils ont de son microworld. Le plaisir de l'humour se construit sur l'expérience de cette connivence. Puis, à ce stade, Dieu demande à l'employée, «Do I need a tray? ». Et le public éclate de rire et applaudit. Izzard vient d'inverser la question devenue anthologique de l'employé de cantine à Darth Vader, «Will you need a tray?». La connexion cognitive chez les spectateurs est cette fois immédiate, car le monde fictionnel a déjà été mis en place : il s'agit de Star Wars version What If dans le microworld d'Eddie Izzard. L'acte cognitif se fait dans un contexte identifié et s'en trouve accéléré d'autant. Ce contexte cognitif est transmédial. La réaction de la salle montre que les spectateurs sont d'accord de suivre l'artiste dans son jeu et son monde fictionnel propre. Le double acte cognitif est pleinement assumé par les deux parties de cet échange conversationnel. Et c'est dans ce contexte très particulier qu'opère l'effet de surprise du gag: Dieu sait pour le plateau et pose donc la question.

C'est ensuite sur cette base qu'Izzard aménage des développements nourris par des informations qui n'existent que dans Star Wars version What If de son microworld, en particulier le fait que les plateaux de la cantine étaient tous mouillés. Quand Darth Vader, dans le sketch original, se résout enfin à aller chercher un plateau, il les passe tous en revue, soulevant chaque plateau l'un après l'autre, et s'exclamant, "This one is wet, this one is wet », etc . Dans la continuation de 2013, l'employée répond à Dieu, « $-N o$, you don't need a tray. Trays have been banned in this canteen. Eight years ago there was a fight and many people died upon the floor. The day was only saved by Mister Stephens, head of catering». Les spectateurs rient et ovationnent le nom de Mister Stephens. "-He did come into the fray and did pick up a tray and immediately it fell out of his hand because it was wet, he picked up another one and that one fell out of his hand because it was wet, a third and a fourth fell out of his hand, the fifth he staple-gunned to his hand ${ }^{13}$, and then he went into action». Pendant cette narration sur le mode pseudo-épique, insérée dans la conversation qui a lieu entre Dieu et l'employée de cantine, Izzard démontre l'action narrée : il fait de manière rudimentaire les gestes de prendre puis de lâcher un plateau l'un après l'autre. Il ne réalise pas complètement l'action, comme le ferait un acteur de théâtre ou de cinéma en plein combat. Ses gestes relèvent de la démonstration dans le sens que lui donne Murphy. Ce ne sont que des indications kinésiques, des suggestions 
suffisantes, qui illustrent le récit qu'il est en train de raconter, exactement comme on pourrait le faire dans une conversation en situation réelle.

Quand Mister Stephens est prêt à sauter dans la mêlée, un plateau agrafé à la main, Izzard, alias l'employée de cantine, mime l'action, produit des onomatopées «Pang, pang!», accompagnées de mouvements de lutte, puis ajoute "Sometimes with the edge bit, petchuk!». Au moment du coup réalisé avec le tranchant du plateau, le geste et l'expression faciale d'Izzard se modifient en même temps que l'onomatopée. La distinction qui est racontée discursivement et kinésiquement est de nature multimodale : elle est visuelle, motrice, sonore et linguistique (par l'onomatopée). Dans un tel moment du récit, le savoir propositionnel et la compétence linguistique des spectateurs sont indissociables de leur intelligence kinésique, grâce à laquelle ils traitent cognitivement et comprennent non seulement le sens des gestes d'Izzard mais aussi la qualité particulière de ses mouvements et de leur modification. Or, les gestes existent en une dimension unique: ils sont réalisés sur scène par Izzard. Mais leur qualité kinésique spécifique est saisie à travers un emboîtement multiple de niveaux ou de plans narratifs et transmédiaux. En effet, je regarde sur ordinateur un lien Internet à YouTube (plan 1), d'Eddie Izzard en conversation performée avec son public (plan 2), montrant son persona dans son microworld raconter une histoire (plan 3), où Dieu parle avec l'employée d'une cantine (plan 4), laquelle raconte à celui-ci l'intervention de Mister Stephens (plan 5) le jour où Darth Vader était venu manger des pâtes (plan 6). Je distingue les plans 5 et 6, car le plan 5 n'existe que dans le spectacle de 2013. Il s'agit d'une continuation de type What Is (informations supplémentaires sur le storyworld original), faite par Izzard à l'égard de son propre récit de type What If, à savoir son sketch sur Star Wars de 2000. Le sketch se poursuit par une conversation entre Dieu et Darth Vader, interrompue par Mister Stephens qui intervient en personne pour la première fois. Nous sommes alors sur le plan 4 (celui de Dieu et de l'employée de cantine). Cette suite est remarquable et mériterait une analyse détaillée. Mais je souhaite terminer par un moment que je trouve particulièrement intéressant pour la question du récit comme acte cognitif.

31 Suite au récit de l'employée de cantine au sujet de l'intervention de Mister Stephens, Izzard fait deux pas pour se repositionner dans le rôle de Dieu, produit un grand inspire sonore, attend deux secondes, puis, en réponse à l'évocation par l'employée de chants en l'honneur des exploits de Mister Stephens, demande, «Such as?».

- And then he went into action, pang, pang! Sometimes with the edge bit, petchuk!

Pang, pang! Even now people sing songs about that day.

[Silence. Un long inspire sonore de Dieu qui fixe l'employée. Deux secondes d'arrêt.]

-Such as?

Ce moment est irrésistiblement drôle pour de multiples raisons ${ }^{14}$. L'une d'entre elles est le timing parfait de l'élocution d'Izzard, qui pousse le spectateur à inférer un temps de suspension réflexive de la part de Dieu ${ }^{15}$. Je souligne que l'inférence ne porte pas sur un éventuel contenu mental ou une représentation, mais bien sur le fait que Dieu s'interroge, qu'il est cognitivement en suspens, d'où son "Such as? ». S'ajoute à cela le choix stylistique de la question. En anglais « Such as? » relève d'un registre linguistique relativement élevé, comme en français «Tel que?", par contraste avec «Comme quoi ?/ Like what?». Associée à une kinésie pondérée, cette question suggère une certaine attention dubitative, une curiosité de Dieu envers l'employée de la cantine, un intérêt pour son récit. Ainsi, la performance d'Izzard génère chez le spectateur un acte cognitif dont le résultat est l'idée d'un acte cognitif chez le personnage incarné par l'humoriste, 
en l'occurrence Dieu, lequel se montre attentif et courtois envers l'employée de cantine. Cette dernière va tout de go se mettre à improviser des chants pseudo légendaires plutôt ratés, en réponse à la question qui lui est posée. Mais Dieu ne fera aucun commentaire désobligeant. Liberté ultime de l'humour qui permet une idée de tendresse, celle que le récit performé d'Izzard fait imaginer de Dieu envers une humaine qui chantonne.

J'espère avoir montré l'intérêt qu'il peut y avoir à prendre pour objet d'analyse un numéro de stand-up posté sur YouTube. Un tel objet réunit des problématiques théoriques d'actualité pour la narratologie, tels le récit transmédial et la narration comme activité en conversation. En outre, un sketch en performance posté sur YouTube donne accès à la temporalité d'actes cognitifs réciproques entre artiste et public, montrant l'importance cognitive du kinésique dans la réception d'une narration en stand-up, ainsi que sa complémentarité forte avec la réalité linguistique du récit.

\section{BIBLIOGRAPHIE}

Allen, Tony (2002), Attitude, Wanna Make Something of It? The Secret of Stand-up Comedy, Glastonbury, Gothic Image Publications.

Baroni, Raphaël (2007), La Tension narrative. Suspense, curiosité et surprise, Paris, Seuil.

Bolens, Guillemette (2012a), The Style of Gestures: Embodiment and Cognition in Literary Narrative, Baltimore, John Hopkins University Press.

Bolens, Guillemette (2012b), “Kinesthetic Empathy in Charlie Chaplin's Silent Films”, in Dee Reynolds \& Matthew Reason, Kinesthetic Empathy in Creative and Cultural Practices, Bristol and Chicago, Intellect, 143-56, [en ligne], URL : http://archive-ouverte.unige.ch/unige:23589

Bolens, Guillemette (2014), "Les simulations perceptives et l'analyse kinésique dans le dessin et dans l'image poétique", Textimage: revue d'étude du dialogue texte-image, [en ligne], URL : http:// archive-ouverte.unige.ch/unige:74799

Dean, Greg (2000), Step by Step to Stand-up Comedy, Portsmouth, Heinemann.

Dynel, Marta (2014), “Participation framework underlying YouTube interaction”, Journal of Pragmatics 73, 37-52.

Herman, David (2003), "Stories as a Tool for Thinking”, in David Herman, Narrative Theory and the Cognitive Sciences, Stanford, CSLI Publications.

Kjærbeck, Susanne \& Birte Asmuß (2005), "Negotiating meaning in narratives: An investigation of the interactional construction of the punchline and the post punchline sequences", Narrative Inquiry 15.1, 1-24.

Matte, Gerard \& Ian McFadyen (2011), "Can we talk? The reframing of social permissions in the comedy of Joan Rivers", Comedy Studies 2.2, 161-71. 
Mittel, Jason (2014), "Strategies of Storytelling on Transmedia Television", in Marie-Laure Ryan \& Jan-Noël Thon, Storyworlds across Media: Toward a Media-Conscious Narratology, Lincoln \& London, University of Nebraska Press, 253-77.

Morreall, John (2009), Comic Relief: A Comprehensive Philosophy of Humor, Oxford, Wiley-Blackwell.

Murphy, Keith M. (2011), "Building Stories: The Embodied Narration of What Might Come to Pass", in Jürgen Streeck, Charles Goodwin and Curtis LeBaron, Embodied Interaction, Language and Body in the Material World. Learning in Doing: Social, Cognitive, and Computational Perspectives, Cambridge, Cambridge University Press, 243-53.

Norrick, Neal R. (2000), Conversational Narrative: Storytelling in Everyday Talk, Amsterdam \& Philadelphia, John Benjamins Publishing Company.

Ochs, Elinor \& Lisa Capps (2001), Living Narrative: Creating Lives in Everyday Storytelling, Cambridge (Mass.), Harvard University Press.

Raskin, Victor (1985), Semantic Mechanisms of Humor, Dordrecht, Reidel.

Ritchie, Chris (2012), Performing Live Comedy, London, Methuen Drama.

Ryan, Marie-Laure (2014), "Story/Worlds/Media: Tuning the Instruments of a Media-Conscious Narratology”, in Marie-Laure Ryan \& Jan-Noël Thon, Storyworlds across Media: Toward a MediaConscious Narratology, Lincoln \& London, University of Nebraska Press, 25-49.

Scarpetta, Fabiola \& Anna Spagnolli (2009), “The Interactional Context of Humor in Stand-Up Comedy", Research on Language and Social Interaction 42.3, 210-30.

Sperber, Dan \& Deirdre Wilson (1986, 1995), Relevance, Communication and Cognition, Oxford, Malden (MA), Victoria (Australia), Blackwell Publishing.

\section{NOTES}

1. Death Star Canteen (https://www.youtube.com/watch?v=Bq03xebtbeU). Force Majeure: Death Star Canteen 2 (https://www.youtube.com/watch?v=SujnUUPs6QU).

2. Sur la relation entre kinésie, cognition et neurosciences, voir Bolens 2012 a \& b, 2014.

3. S'ajoute, d'ailleurs, à cette troisième transmédialisation, le premier sketch en version lego, où les personnages et les décors sont entièrement faits de legos, la bande son restant celle de la performance live d'Eddie Izzard: https://www.youtube.com/watch?v=Sv5iEK-IEzw. Concernant les nouvelles formes d'interaction et les niveaux de communication sur Internet et en particulier YouTube, voir Dynel 2014.

4. Le terme travesti ne suffit pas à donner une idée exacte du style vestimentaire d'Izzard, qui est toujours totalement personnel, évolutif, et inclassable.

5. Pour Matte et McFadyen, «Stand-up comedy ... differs from conventional dramatic performance in that it resembles, at least superficially, a conversation between the performer and the audience. The audience is not merely witnessing a conversation between characters but is treated as an active player in the scene, albeit one with a particularly defined role " (2011: 163). Voir aussi Scarpetta \& Spagnolli.

6. Ce genre de question correspond à ce que Ochs et Capps appellent «story preface ». Dans une conversation, cette préface au récit sert à piquer l'intérêt des interlocuteurs et à les préparer à une histoire d'une certaine durée, plus longue qu'un tour de parole normal (Ochs \& Capps 2001 : 117-118). 
7. Dean reprend les analyses de Victor Raskin (1985), mais remplace la notion de script 1 puis 2 , par celle de récit (story) 1 puis 2 . Sur les questions du « joke as narrative » et de la relation «buildup, pivot, punchline », voir Norrick 2000: 170ff. Sur la punchline, voir aussi Kjærbeck \& Asmuß.

8. Voir Morreall (2009: 101-110), dont les analyses permettent de comprendre pourquoi les sketchs qui incitent à la haine sont graves. Ce n'est jamais le cas dans les spectacles d'Izzard, bien au contraire.

9. Sur la surprise en narration, voir Baroni (2007).

10. « Il est chef/manager du service en cantine ».

11. "Moreover, narrative structures are almost exclusively analysed along verbal linguistic features. Considering that narrative studies emerged through the analysis of literary texts, this makes some sense. However, importing narrative analysis into the study of face-to-face interaction requires close attention to the multimodal construction of the particular conversational forms in which narratives appear » (Murphy 2011: 244)

12. Sur l'interaction multimodale, voir l'ensemble du volume dans lequel est publié l'essai de Murphy, édité par Streeck, Goodwin et LeBaron, ainsi que le numéro spécial du Journal of Pragmatics 46, 2013, dédié à l'analyse conversationnelle et l'interaction multimodale.

13. «Il fixa le cinquième plateau à sa main au moyen d'un pistolet à agrafes ».

14. Je l'affirme en étant consciente du fait que la relation d'humour est de nature esthétique et donc subjective.

15. Sur l'importance cruciale du timing dans la comédie de stand-up, voir Dean (2000: $125 \mathrm{ff}$.) et Tony Allen (2002: 19).

\section{RÉSUMÉS}

Un comédien ou une comédienne de stand-up est une personne dont l'action consiste à se tenir devant des spectateurs et à raconter des histoires pour les faire rire. Le genre du stand-up peut être défini comme une conversation performée entre le comédien et les spectateurs. La réussite de cet échange se mesure à un impacte cognitif chez les spectateurs, qui se manifeste par leurs sourires, leur rire répété, voire leur hilarité. Je propose de prendre pour sujet d'analyse deux numéros du grand humoriste anglais Eddie Izzard. Ces deux sketchs sont postés sur YouTube et réunissent des problématiques théoriques d'actualité pour la narratologie, tels le récit transmédial et la narration comme acte cognitif en conversation. Un récit performé dans un numéro de stand-up posté sur YouTube donne accès à travers sa médiatisation à la temporalité d'actes cognitifs réciproques entre artiste et public, et montre l'importance cognitive du kinésique dans la production et la réception d'une narration, ainsi que la complémentarité forte de cet aspect avec la dimension linguistique de l'histoire racontée.

A stand-up comedian is a person whose action consists in standing on stage to trigger laughter by telling stories. Stand-up comedy can be defined as a performed conversation between a comedian and her audience. The measure of its success is a cognitive impact that manifests itself in the audience's smiles, reiterated laughter, even hilarity. In this article I analyse two comedy acts by the famous British actor Eddie Izzard. Both acts are posted on YouTube and prove relevant to theoretical issues of interest to narratology today, i.e., transmedial storytelling, and the cognitive acts of narratives in conversation. A performed narrative in a stand-up comedy posted on YouTube gives access via its mediatisation to the temporality of reciprocal cognitive acts 
between comedian and audience, showing the cognitive relevance of kinesis in the production and reception of narrative, matching in importance the linguistic dimension of storytelling.

INDEX

Mots-clés : actes cognitifs, récit transmédial, narration en conversation, la comédie de stand-up, blagues, gestes et kinésie dans l'acte de raconter, Eddie Izzard

Keywords : cognitive acts, transmedial storytelling, narrative in conversation, stand-up comedy, jokes, gestures and kinesis in storytelling, Eddie Izzard

\section{AUTEUR}

\section{GUILLEMETTE BOLENS}

Université de Genève 